adiposity, were measured directly. Study participants were traced, mailed a health questionnaire in 1962/1966 (mean age 45.1 years) which included enquiries regarding self-reported height and weight, and were followed for subsequent mortality experience - with $>$ $99 \%$ completion - until the end of 1998. BMI was categorised into quartiles in the present analyses.

Setting: USA.

Participants: 14638 men enrolled in Harvard University in the given years, who completed the subsequent health questionnaire, and whose vital status could be ascertained.

Main Outcome Measure: CHD death.

Results: Over a maximum of 82.5 years of follow-up (median 56.5 years), there were 1401 deaths from CHD. Following adjustment for age and other CHD risk factors (cigarette smoking, physical activity, blood pressure) at college entry, relative to the lowest weight quartile (mean $\mathrm{BMI}=18.7 \mathrm{~kg} / \mathrm{m}^{2}$ ), there was an elevated risk of CHD mortality in men in the highest quartile (mean $\mathrm{BMI}=25.0 \mathrm{~kg} / \mathrm{m}^{2}$; hazards ratio $1.28,95 \%$ CI 1.10 to 1.49 ) but not the intermediate groups. Following additional control for BMI in middle-age, this increased CHD risk in the highest quartile was eliminated $(1.03 ; 0.87$ to 1.21$)$.

Conclusion: In this cohort, higher BMI in early adulthood was associated with an elevated risk of CHD mortality several decades later but this effect appeared to be entirely meditated via BMI in middle-age.

\section{THE LONG TERM EFFECTS OF CHILDBEARING AND BREASTFEEDING ON BODY MASS INDEX IN MIDDLE AGED WOMEN RESULTS FROM THE MILLION WOMEN STUDY}

${ }^{1} \mathrm{~K}$ Bobrow, ${ }^{2} \mathrm{M}$ Quigley, ${ }^{3} \mathrm{~J}$ Green, ${ }^{3} \mathrm{G}$ Reeves, ${ }^{3} \mathrm{~V}$ Beral, on behalf of the Million Women Study Collaborators ${ }^{1}$ Department of Public Health, University of Oxford, Oxford, UK; ${ }^{2}$ National Perinatal Epidemiology Unit, University of Oxford, Oxford, UK; ${ }^{3}$ Cancer Epidemiology Unit, University of Oxford, Oxford, UK

\section{doi:10.1136/jech.2009.096727d}

Objectives: To examine the relation between childbearing and breastfeeding and subsequent body mass index (BMI) in middleaged women.

Design: Cross-sectional analysis within a large prospective study. Participants: One million UK women recruited into the Million Women Study during 1996-2001. Participants completed questionnaires collecting information on personal and lifestyle characteristics, medical and reproductive history including questions on childbearing and breastfeeding, anthropometry, physical activity, and socio-demographic in factors.

Main Outcome Measures: Mean BMI stratified by parity and breastfeeding and adjusted for age, geographical region, socioeconomic status, smoking status, strenuous physical activity, age at first birth, and years since last birth.

Results: 980474 women were included in the main analysis. $87 \%$ of the study population were parous, mean parity was 2.1 (1.2) and $68 \%$ of parous women ever breastfed. The mean lifetime duration of breastfeeding per child was 3.8 (SD 3) months. Mean BMI increased significantly with each birth from 25.8 (95\% CI 25.7 to 25.8) for nullips to 28.1 (95\% 28.1 to 28.3 ) for women with five or more births. Parous women who breastfed had significantly lower BMIs than their nonbreastfeeding counter parts. The effect was attenuated by adjusting for socio-economic status but remained significant even after full adjustment The mean BMI decreased as lifetime duration of breastfeeding increased: compared with women who never breastfed, mean BMI decreased by $0.8(95 \%$ CI 0.71 to 0.82$) \mathrm{kg} / \mathrm{m}^{2}$ in those with a lifetime breastfeeding duration of more than 9 months. This relationship was statistically significant $(\mathrm{p}<0.0001)$ and maintained at each parity level. Conclusions: In this analysis including one million middle-aged women in the UK we found that BMI increased with increasing parity, but that this increase would be offset if women breastfed. These relationships were independent of socioeconomic status, geographic region, smoking, exercise, age at first birth and time since last birth. These findings contribute to the body of evidence that childbearing and breastfeeding have sustained long term effects on the health status of women.

\section{Ethnicity and young people}

\section{EXPLAINING THE MENTAL HEALTH ADVANTAGE OF BRITISH INDIAN CHILDREN}

A Goodman, V Patel, D Leon. Department of Epidemiology and Population Health, London School of Hygiene and Tropical Medicine, London, UK

\section{doi:10.1136/jech.2009.096727e}

Objective: To investigate the causes of the lower rate of mental disorders diagnosed in British Indian children compared to White children.

Design: Secondary analysis of two population-based, crosssectional surveys of child mental health (the British Child and Adolescent Mental Health Surveys of 1999 and 2004).

Setting: Great Britain (nationally-representative sample). Mental health outcome measures: Parent Strengths and Difficulties Questionnaire (SDQ); teacher SDQ; child SDQ; multi-informant clinical diagnosis.

Participants: 16449 White and 419 Indian children aged 5-16. The detailed multivariable models focused on the 13868 White and 361 Indian children from England whose parents had completed an $\mathrm{SDO}$ in English.

Results: There was a substantially lower prevalence $(p<0.001)$ of any mental disorder in Indians (3.4\%, 95\% CI 1.9 to 5.9) compared to Whites was $(9.6 \%, 95 \%$ CI 9.1 to 10.2$)$. Strong evidence $(p<0.002)$ of an Indian advantage for externalising problems/disorders was consistently observed for the parent, teacher, child SDQs and for clinical diagnosis. Detailed psychometric analyses of the SDO and clinical interview measures provided no evidence that this Indian mental health advantage could be explained by a measurement bias in the assessment of mental health. In multivariable analyses the unexplained difference between Indians and Whites decreased somewhat after adjusting for the fact that Indian children were more likely to live in two-parent families (92.2\% vs. $65.4 \%$ ) and less likely to have academic difficulties (e.g. $2.9 \%$ vs. $8.6 \%$ for parent-reported learning difficulties). In models adjusting for a larger number of child, family, school and area variables the difference reduced only by about a quarter (e.g. from 1.08 to $0.75 \mathrm{SDQ}$ points on the parent SDQ) and remained highly significant $(p<0.001)$. There was little or no evidence of an ethnic difference for internalising problems/disorders in unadjusted or adjusted models.

Conclusions: The mental health difference between Indian and White children is specific to a substantial advantage for externalising disorders, and this advantage appears to be real rather than due to a reporting bias. This advantage is largely unexplained by major risk factors for child mental health problems available in this dataset. Further qualitative and quantitative research into the causes of this advantage has the potential to yield insights which could improve the mental health of children of all ethnic groups.

\section{EMERGENCE OF ETHNIC DIFFERENCES IN BLOOD PRESSURE IN ADOLESCENCE: THE DETERMINANTS OF ADOLESCENT SOCIAL WELL-BEING AND HEALTH LONGITUDINAL STUDY}

${ }^{1} \mathrm{E}$ Lenguerrand, ${ }^{1} \mathrm{~S}$ Harding, ${ }^{1} \mathrm{M}$ Whitrow, ${ }^{1} \mathrm{M}$ Maynard, ${ }^{2} \mathrm{~K}$ Cruickshank, ${ }^{1} \mathrm{G}$ Der, ${ }^{1}$ A Teyhan. ${ }^{\prime}$ Medical Research Council, Social and Public Health Sciences Unit, University of Glasgow, Glasgow, UK; ${ }^{2}$ Division of Cardiovascular and Endocrine Sciences, University of Manchester, Manchester, UK

doi:10.1136/jech.2009.096727f

Objective: To examine ethnic differences in changes in Blood Pressure (BP) between early and late adolescence in the UK. 